\title{
28 Research Soure \\ Longitudinal Prediction of Motor Dysfunction After Stroke: A Disconnectome Study.
}

\author{
Lia Talozzi \\ University of Bordeaux \\ Valentina Pacella \\ University of Bordeaux \\ Maurizio Corbetta \\ University of Padova \\ Stephanie J Forkel \\ University of Bordeaux \\ Michel Thiebaut de Schotten \\ University of Bordeaux
}

Lilit Dulyan ( $\square$ lidulyan@gmail.com )

University of Bordeaux https://orcid.org/0000-0002-8652-7828

\section{Research Article}

Keywords: disconnectome, stroke, motricity, longitudinal, recovery, plasticity

Posted Date: December 30th, 2021

DOI: https://doi.org/10.21203/rs.3.rs-1131626/v1

License: (c) (i) This work is licensed under a Creative Commons Attribution 4.0 International License.

Read Full License 


\section{Abstract}

Motricity is the most commonly affected ability after a stroke. While many clinical studies attempt to predict motor symptoms at different chronic time points after a stroke, longitudinal acute-to-chronic studies remain scarce. Taking advantage of recent advances in mapping brain disconnections, we predict motor outcomes in 62 patients assessed longitudinally two weeks, three months, and one year after their stroke. Results indicate that brain disconnection patterns accurately predict motor impairments. However, disconnection patterns leading to impairment differ between the three time points and between left and right motor impairments. These results were cross-validated using resampling techniques. In sum, we demonstrated that while some neuroplasticity mechanisms exist changing the structure-function relationship, disconnection patterns prevail when predicting motor impairment at different time points after stroke.

\section{Introduction}

Stroke is one of the most significant disorders worldwide both in terms of mortality and long-term disabilities (Feigin et al. 2021). Despite considerable research into the risks and treatments of cerebrovascular diseases (Gurol and Kim 2018), every 3 seconds, someone suffers a stroke, resulting in various cognitive and motor dysfunctions (www.world-stroke.org). Motricity is understood as a motor impulse sent efferently down a nerve toward a muscle. This motor function is the most commonly affected ability after stroke ( 88\%, Aqueveque et al. 2017). Motor deficits post-stroke occur secondary to a vascular rupture (haemorrhage) or an occlusion (ischemia) leading to decreased blood flow in one of the arteries that supply the motor network in the brain. This network includes the primary motor and somatosensory cortices, premotor regions in frontal and parietal cortex, and the basal ganglia. The main outflow tract is the corticospinal tract (CST, Bhuiyan et al. 2014) that descends from the cortex through the internal capsule, the cerebral peduncle, the brainstem down into the spinal cord. Post-stroke motor deficits such as partial paralysis or muscular weakness (hemiparesis) of the upper (e.g. hands, arms) or lower extremities (e.g. legs, foot) are present in about $88 \%$ of stroke patients at the acute stage. Longitudinally, merely $12 \%$ of patients demonstrate a full functional recovery after rehabilitation (Aqueveque et al. 2017). Although several rehabilitation programmes have been developed (e.g., The Queen Square Upper Limb (QSUL) Neurorehabilitation programme, Kelly et al. 2020), at present, there is no reliable prediction of recovery. This absence of reliable predictions can lead to frustration and reduced motivation in patients and family members, which is unfavourable to rehabilitation success (Kusec et al. 2019). Since proper and early interventions are crucial for a successful recovery (Coleman et al. 2017), early recovery prediction will likely help clinicians adjust their rehabilitation efforts to significantly improving motor functioning after a stroke. Hence, accurate predictions may result in more efficient and successful recovery of patients long-term.

Using various types of biomarkers, a wealth of clinical studies attempted to predict motor symptoms at the acute (first seven days), subacute (within one week to three months), and early and late chronic phases (starting at three months after onset) after stroke (Boyd et al. 2017; Connell et al. 2018; Stinear 
2017; Lin et al. 2018). This body of work showed that the quality of motor abilities after stroke is associated with i) the severity in the clinical behavioural baseline scores assessed at the acute stage (Nijland et al. 2010), ii) the presence and amplitude of motor evoked potentials triggered with transcranial magnetic stimulation (TMS, Feys et al. 2000), iii) the volume and location of the lesion calculated with structural magnetic resonance imaging (Schiemanck et al. 2006), iv) the structural integrity of the corticospinal tract detected with diffusion-weighted imaging (DWI, Puig et al. 2011; Stinear 2017), task-related cortical activity and resting-state functional connectivity measured with functional MRI (fMRI, Rehme et al. 2011; Thiel and Vahdat 2015; Carter et al. 2010; Carter et al. 2012). Although most of these studies demonstrate a strong statistically significant relationship between behavioural motor scores and distinct biomarkers that can explain up to $96 \%$ variance of the data (Granziera et al. 2012), they do not validate the results of their models in an independent sample of patients which reduces the ecological validity and generalisability of these results. A systematic review (Kim and Winstein 2017) showed that only 8 out of 71 studies aiming to predict motor outcomes performed a resampling technique (e.g. $\mathrm{k}$-fold crossvalidation) to evaluate their models. These methods fight against a common problem in machine learning known as overfitting. A model perfectly describes the known data - training set, but it performs poorly on new observations - validation set (Ying 2019). Those algorithms that demonstrate the perfect ability to capture the variability in data without applying resampling methods may fail to generalise as a universal model to predict recovery (Heil et al., 2021). Thus, testing the model for out-of-sample predictions is crucial for translating the knowledge from research to a clinically useful tool to maximise patient benefits. As such, the absence of externally validated models makes their performances unreliable in predicting motor outcomes of new individual patients (Berrar, 2019) and further high-quality statistic studies are required in order to identify reliable brain markers for motor deficits after a stroke.

Many efforts have been dedicated to studying lesion localisation and associating it with motor disabilities (see review Schiemanck et al. 2006). It is undeniable that these symptoms can also occur with lesions to the grey or white matter (Catani and ffytche 2005; Carrera and Tononi 2014; Thiebaut de Schotten and Foulon 2018; Thiebaut de Schotten et al. 2008). For example, in spinal cord disconnections, the information processed in the intact motor cortex cannot be conducted to the relevant part of the body, causing motor paralysis (Tidoni et al. 2015). In the 19th century, this type of interruption of motor function distant from a lesioned region was discussed by a pioneering neurologist, Constantin von Monakow (1853-1930, von Monakow 1914), and referred to as diaschisis cerebrospinalis (Finger et al. 2004). However, a proper statistical measure of this theoretical concept was not possible thus far.

Advanced neuroimaging methods, including DWI that measures water displacement in brain tissue, opened a new avenue into exploring white matter connections (Moura et al. 2019; Catani and Thiebaut de Schotten 2008). Nowadays, the lesion load (the proportion of destruction) in the CST is one of the most popular predictors of motor disabilities and is interpreted as a measure of diaschisis (Carrera and Tononi 2014; Koch et al. 2021). However, lesion load in the CST does not account for the dysfunction of other distant brain regions. In other words, lesion load analysis is not an accurate operationalisation of diaschisis (Thiebaut de Schotten et al. 2014). Limiting our analysis to the lesion location and neglecting possible interruptions of functions in other brain structures might miss valuable information to 
understand behavioural outcomes after stroke. The disconnection analysis has been proposed recently as a possible solution to this problem (Foulon et al. 2018; Kuceyeski et al. 2013). Disconnection analyses consider a probability of dysfunctions in other brain areas. For example, if a patient has a lesion in a subcortical region (e.g. posterior limb of the internal capsule), the disconnection analysis will provide us with a probability of interruption of the white matter connection (e.g. CST) that links the subcortical (internal capsule) and cortical regions (e.g. primary motor cortex). When comparing those methods, the disconnection analysis often explains more variance in data than lesion load analysis (Corbetta et al. 2015; Hope et al. 2016). Thus, prediction studies may benefit from the redirection of the focus in clinical research from lesion localisation analysis to the analysis of the disconnections where remote alterations of a focal damage to distant regions can also be considered as a contributor to a network of structures and functions.

Another issue in the literature that may downplay the full potential of predicting motor symptoms is the considerable variation in motor assessments. Each clinical test utilised in symptom prediction studies focuses only on one aspect of motor dysfunction (Kim and Winstein 2017), which is not capturing the whole complexity of motor disabilities (Cheung et al. 2012), making both the prediction and comparisons between studies challenging. Corbetta et al. (2015) partially overcame this limitation by applying a principal component analysis that explained a great majority of variability $(77 \%)$ across all motor tasks and patients with just two components (i.e. left and right side of the body). Extracting common patterns of motor outcomes from different assessments and eliminating the redundancy in the data should increase the performance of predictive models in the future (Corbetta et al. 2015).

Recent work considered the main limitations outlined above and conducted a ridge regression model that automatically eliminates irrelevant predictors by applying second-order penalty terms (i.e. L2normalisation) to avoid overfitting. This model explained up to $37 \%$ and $28 \%$ of the variability in motor outcomes at the subacute stage for left motor recovery (i.e. right hemisphere lesion) and right motor recovery (i.e. left hemisphere lesion), respectively (Salvalaggio et al. 2020). While these results are encouraging, the predictions reported are limited to 2 weeks after the stroke and do not extend to the longterm prognosis of motor abilities.

This study aimed to predict acute-to-chronic motor outcomes in 62 stroke patients two weeks, three months, and one year after stroke from the lesion defined at the acuate stage (two weeks post stroke). Using a cross-validation longitudinal study design and a disconnection analysis should overcome the limitations of current predictive models in stroke. We modelled the pattern of brain disconnections based on the Disconnectome (Thiebaut de Schotten et al. 2020), which characterises brain disconnections along 46 anatomical dimensions. The validation of the predictions was calculated using hierarchical linear regression models with cross-validation resampling and yielded medium to large effect sizes.

\section{Methods}

\section{Participants}


Sixty-two patients (age: $M=53.7, S D=10.7$, range 22- 83 years; 34 males; 57 right-handed, see Table 1) met the inclusion criteria: All patients were older than 18 years, presented with first-ever ischemic (83\%) or haemorrhagic $(17 \%)$ stroke and behavioural deficits as assessed by a neurological examination. Patients who had a history of neurological or psychiatric presentations (e.g. transient ischemic attack), multifocal or bilateral strokes, or had MRI contraindications (e.g. claustrophobia, ferromagnetic objects) were excluded from the analysis $(n=131$ patients, see the enrollment flowchart in the supplementary materials from Corbetta et al. 2015). We further limited our analysis to the patients whose motor functions were systematically assessed at 2 weeks, 3 months, and 1 year after their stroke for optimal longitudinal comparisons. This study was approved by the Washington University in Saint Louis Institutional Review Board and all participants gave their signed informed consent.

Table 1 Demographics of patients

\section{Statistic Min Max}
Age (mean/SD)
$53.73 / 10.66 \quad 22$
83

Education (mean/SD) $\quad 13.66 / 2.76 \quad 5 \quad 20$

Handedness (\% right-handed) 91.94

Sex (\% female) $\quad 45.16$

\begin{tabular}{lllc} 
& Statistic & Min & Max \\
\hline Age (mean/SD) & $53.73 / 10.66$ & 22 & 83 \\
\hline Education (mean/SD) & $13.66 / 2.76$ & 5 & 20 \\
\hline Handedness (\% right-handed) & 91.94 & & \\
\hline Sex (\% female) & 45.16 & &
\end{tabular}

Abbreviations: SD = standard deviation

Motor abilities assessment

Motor functions were examined for the upper and lower extremities. For upper extremities, active range of motion against gravity was measured by goniometer at shoulder flexion and wrist extension (Dreeben 
2008). During the examination of shoulder flexion, patients are asked to raise their arm against gravity as high as possible. The movement amplitude is recorded as the angle between the goniometer centred on the shoulder and the lateral torso. The wrist extension examination requires patients to sit with their arm on the table in a resting position with their palms down, and they are asked to bend back their wrist against gravity. Wrist extension is measured as the angle between the goniometer centred on the wrist and the forearm.

Grip strength was measured using a dynamometer (Demeurisse et al. 1980). Each patient's examined arm was placed with the elbow flexed at $90^{\circ}$. Their fingers flexed for a maximal contraction over the dynamometer handle, while the forearm and wrist were kept in a neutral position. The patients were asked to take a breath while exerting the maximum grip effort during three consecutive trials. The strength score is recorded in kilograms, and the total score is calculated as the average over three trials (Fess and Moran 1981).

The patients' dexterity and bimanual coordination was measured with the 9-Hole Peg Test (pegs/second) assessing gross movements of arms, hands, and fingers, and fine motor extremity. The test includes a one-piece board with a concave folded dish containing nine pegs next to a 9-holes matrix for the pegs. The task instructions require patients to place and remove the nine pegs one at a time and in random order as quickly as possible (Mathiowetz et al. 1985; Oxford Grice et al. 2003). The final score is calculated as the time in seconds elapsed from the touch of the first peg to when the last peg is placed back into the dish.

The Action Research Arm Test (ARAT, van der Lee et al. 2001) assesses the ability to perform purposeful movements with the upper limb extremities. Patients had to grasp, grip, pinch objects of different weights and shapes and perform gross motor movements. The ARAT's four subtests have 19 items in total. Each item is rated on a four-point scale (0-3) where higher scores indicate better performance. If the patient scores $<3$ on the first item, the examiner advances to the second item two (the most accessible item). If

the score for the second item is 0 , the rest of the items will automatically be scored as 0 for and the test is stopped. If the patients score $<3$ on the first item but $>0$ on the second item, the remaining items are administered (Lyle 1981).

For lower extremities, a combined walking index (Kempen et al. 2011; Perry et al. 1995), left/right total motricity index, ankle dorsiflexion goniometry for left/right active range of motion against gravity was recorded (Dreeben 2008).

\section{MRI acquisition and preprocessing}

\section{MRI scan acquisition}

Neuroimaging was performed on a Siemens 3T Tim-Trio scanner at the School of Medicine of the Washington University in St. Louis. All structural scans were collected 2 weeks after the stroke and included (1) a sagittal MP-RAGE T1-weighted image (repetition time $=1950 \mathrm{msec}$, echo time $=2.26 \mathrm{msec}$, 
flip angle $=9$ degrees, voxel size $=1.0 \times 1.0 \times 1.0 \mathrm{~mm}$, slice thickness $=1.00 \mathrm{~mm}) ;(2)$ a transverse turbo spin-echo T2-weighted image (repetition time $=2500 \mathrm{msec}$, echo time $=435 \mathrm{msec}$, voxel-size $=1.0 \times 1.0 \mathrm{x}$ $1.0 \mathrm{~mm}$, slice thickness $=1.00 \mathrm{~mm}$ ); and (3) a sagittal FLAIR (fluid attenuated inversion recovery) (repetition time $=7500 \mathrm{msec}$, echo time $=326 \mathrm{msec}$, voxel-size $=1.5 \times 1.5 \times 1.5 \mathrm{~mm}$, slice thickness $=$ $1.50 \mathrm{~mm})$.

\section{Lesion segmentation (native space)}

Lesions were manually segmented on the T1-weighted MRI images using the Analyze biomedical imaging software system (Robb and Hanson, 1991). Two board-certified neurologists (Drs Corbetta and Carter) reviewed all segmentations blinded to the individual behavioural data.

\section{Spatial normalisation (MN/152)}

To align T1-weighted MRI scans of patients to a standard stereotaxic space (Montreal Neurological Institute space, MNI152 Grabner et al. 2006), it is necessary to first address the issue of space deformation caused by brain lesions during spatial normalisation (Brett et al. 2001; Ripolles et al. 2012; Volle et al. 2013). An enantiomorphic approach was implemented in the current data analysis: the nativespace lesions were replaced with healthy tissue of the same region in the contralateral hemisphere (Nachev et al. 2008). Subsequently, affine and diffeomorphic deformations were applied to co-register scans and lesions to the MNI152 space using the Advanced Normalization Tool (ANTS, Avants et al. 2011; Klein et al. 2009). These analyses are available as part of the 'Normalisation' part of BCBtoolkit (Foulon et al. 2018; http://toolkit.bcblab.com).

\section{Generation of disconnection maps.}

Methodological details are available from Thiebaut de Schotten et al. (2020). In brief, each lesion serves as the input for the BCBtoolkit's Disconnectome tool that computes maps of white matter pathway disconnection probabilities and its impact on loss of function (Foulon et al. 2018; http://toolkit.bcblab.com). Probabilities of white matter pathways were derived from a normative population of 163 healthy controls ( $44.8 \%$ males) using a diffusion-weighted imaging dataset acquired on a 7T scanner as part of the Human Connectome Project (Vu et al. 2015). The pattern of brain areas that were disconnected in each stroke was subsequently characterised by measuring the average level of disconnection in subcortical areas and areas derived from a multimodal atlas of the brain surface (Glasser et al. 2016). Subcortical areas included manually defined by MTS and included the thalamus, the putamen, the pallidum, the hippocampus, the caudate nucleus, and the amygdala. Hence, for each of the 62 patients in this study, the disconnection probability of 372 grey matter structures (186 structures in each hemisphere: 180 cortical and 6 subcortical) was obtained.

Dimensionality reduction with Principal Component Analysis

\section{Behavioural components}


To summarise behavioural measurements data while keeping as much variability as possible and minimising noise, behavioural components were extracted as described in Corbetta et al. (2015). In brief, an oblique rotation principal component analysis was applied to the motor scores obtained with different neuropsychological tests described above. Two components (left and right side of the body) resulted from the analysis explaining $77 \%$ of the observed variance in the data. The analysis was computed in Matlab (MathWorks Inc.).

\section{Disconnection map components}

The Disconnectome maps components consist of 46 components, where 30 components have already been shown to capture more than $90 \%$ of the variance in the distribution of disconnection maps in stroke (Thiebaut de Schotten et al. 2020). These components were derived from an independent normative dataset of 1333 disconnection maps of ischemic stroke patients $(M=63.89, S D=15.91$, range 18-97 years; $56.1 \%$ males) fully described in (Xu et al. 2018) and (Thiebaut de Schotten et al. 2020).

We used the Disconnectome 46 components to decode the profile of disconnection in our sample of 62 patients. Linear regression with 46 independent variables (i.e. the disconnectome components) was performed in RStudio (v.1.3.1093; RStudio Team, 2020) to predict the disconnection patterns for each patient. In doing so, beta coefficients for each predictor corresponded to the component's scores of each patient. Adjusted r-squared values represented the percentage of each patient's disconnection map variance explained by the 46 components.

The linear regression analysis demonstrated that the Disconnectome (46 principal components) was able to capture the variance in the disconnection patterns of each patient from the longitudinal dataset. The mean adjusted $r$-squared across the group is 0.94 , with a standard deviation of 0.076 .

\section{Hierarchical linear regression analyses}

The analyses for the anatomical prediction of the motor outcome were carried out in RStudio (v.1.3.1093; RStudio Team, 2020).

Six hierarchical linear regression analyses were performed. Each hierarchical linear regression used the patients' estimated scores of each component (i.e. beta coefficients from the linear regression) to predict two principal components of motor scores (the left and right side of the body, $n=2$ ) two weeks, three months, and one year after the stroke ( $n=3$ time points). The least contributively predictor was removed iteratively by the hierarchical linear regression. The goodness of fit of consecutive linear models was compared statistically using an F-test. The process was repeated until no significant difference could be identified between two consecutive models.

This procedure is available as supplementary code with the manuscript (see https://github.com/lidulyan/Hierarchical-Linear-Regression-R-). 
To avoid inflation of the significance of our results (i.e. overfitting), the hierarchical linear regression analyses were performed on $78 \%$ of the original dataset (training set).

To achieve the generalisability of our model and avoid overfitting, which is one of the limitations of currently available predictive models in the stroke literature, the optimised model with the reduced number of predictors was tested on the unseen by the model data ( $22 \%$ from the original dataset). This process was repeated 5000 times with different data splits by varying the seed randomly to control the potential error induced by the split. A median from the R-squared distribution, representative of the most representative model, was used to describe model fitness.

Disconnectome components with highly significant contributions to motor impairment $(p<0.001)$ were displayed using Surfice (https://www.nitrc.org/projects/surfice/).

\section{Anatomy}

The white matter was identified and labelled manually by expert anatomists (MTS/SJF) according to the Atlas of Human Brain Connections (Rojkova et al. 2016; Thiebaut de Schotten et al. 2015).

\section{Results}

Lesion characteristics (location, overlay)

Patients' lesions and disconnection maps from the dataset $(\mathrm{N}=62)$ were distributed bilaterally with $67 \%$ of lesions in the right hemisphere (Figure 1). The most common site of damage was observed in the right subcortical regions ( $n=27$; other areas $n<15)$, including the thalamus, putamen, caudate, pallidum, hippocampus, amygdala, nucleus accumbens, insula, subcallosal cingulate, paracingulate, and parahippocampal areas. The disconnection maps generated with the individual lesions mirrors this bilateral but right prevalent pattern of disconnection with the highest overlap involving the ventral visual pathways, internal capsule, and perisylvian white matter (Catani and Thiebaut de Schotten 2012).

\section{Behavioural characteristics (Motor impairment)}

Figure 2 indicates that a significant portion of patients in our sample presented with motor impairments (motor score $<0$ ). The proportion of patients with motor disabilities reduced from two week to one year after the stroke in line with previous findings (Hatem et al. 2016).

\section{Prediction of the motor outcomes}

Hierarchical linear regressions used the patients' disconnectome components to predict motor scores' components (the left and right side of the body, $n=2$ ) two weeks, three months, and one year after the stroke ( $n=3$ time points).

Each hierarchical linear regression was repeated 5000 times with different training and testing dataset splits. Figure 3 shows that the models' prediction based on the hierarchical linear regression analysis is 
more consistent at two weeks than three months or one year, both for the left and right sides of the body.

Notably, the ability of the models to explain the variability in motor outcomes on the training dataset was higher than in the testing data. For example, the prediction accuracy of right motor scores a year after the stroke reaches $64 \%$ accuracy ( $p<0.001$; Figure 4$)$ on the training set and $\sim 4 \%(p=0.471$; Figure 4$)$ on unseen data.

The number and the type of components retained in the model vary across the affected hemispheres and periods.

The variability in brain disconnections related to motor performance is particularly evident for the right hemibody, as illustrated in Figure 5 for highly significant components $(p<0.001)$. Motor disorders at 2 weeks after stroke were mainly associated with the disconnection of the component corresponding to the left corticospinal tract. However, the contributions changed at three months involving premotor and superior parietal connectivity and prefrontal lobe and frontotemporal connections (i.e. arcuate fasciculus). Eventually, one year after the stroke, sensory-motor short U-shape fibres appear to predict individual motor scores. In contrast, left hemibody motor performance was systematically associated with white matter connections between the frontal, temporal, and insular cortices, except for the supramarginal gyrus in the inferior parietal lobe that contributed significantly one year after the stroke.

\section{Discussion}

In this study, we predicted motor impairments across different time points based on the pattern of acute brain disconnections two weeks after a stroke. Our analysis reveals three primary results. First, brain disconnection patterns can accurately predict motor impairment. Second, the disconnection patterns leading to impairment are not the same at the two weeks, three months, and one year after a stroke. Third, the predictions were replicable, to some extent, in the cross-validation analysis. Overall, the results indicate that while some plasticity mechanisms exist changing the structure-function relationship, early disconnection patterns prevail when predicting motor impairment at different times after the stroke.

Prediction of behavioural impairments based on brain data has been one of the early goals of clinical neuropsychology. Pioneer scientists analysed neuropsychological impairments of single cases and tried to link them with brain lesions (e.g. patient Leborgne with a lesion in the left inferior frontal gyrus and loss of articulation). While this approach has offered fruitful discussions on the link between a specific region in the brain and a cognitive function (i.e. localisationism), it is hard to use this approach to accurately predict individual patients in the clinic because of variations in lesion characteristics and a priori anatomical variations. These structural and functional differences in healthy brains add extra variability and, therefore, are an additional challenge to generalise a single case to the whole patient population (Smith et al. 2019). Another extremity of analysing lesion-symptom relationships is the grouplevel analysis, where all data is averaged to a common space. This diminishes the role of interindividual variability (Forkel et al. 2021), and inference about an individual based on group-level data analysis leads to a potential error, known in the literature as "ecological fallacy" (Portnov et al. 2007; Robinson 1950). 
Therefore, predictions based on this approach could be misleading. With the availability of big datasets and the development of new statistical tools, it became possible to advance from single cases and grouplevel studies toward an integrative approach that accounts for interindividual variations but generalises to the group-level. This integration allows researchers to make personalised and individual predictions like in the present study.

Preliminary attempts used different machine learning approaches to predict motor impairment. However, most of the studies fall into one of the pitfalls that were described above (i.e. overfitting, generalisability, and diaschisis) as the models are not validated in an independent dataset. They also focus on the lesion location, statistically ignoring the remote effects of a lesion on distant brain regions. Finally, they only consider a few aspects of motor functions independently rather than the entire pattern of motor abilities. Akin to Salvalaggio et al. (2020), we considered these issues that may downplay the potential power of prediction motor symptoms based on neuroimaging data. We applied the resampling method to validate the results. We used behavioural data that includes excellent variability of neuropsychological tests that capture motor disorders from different angles. We applied disconnection analysis that is a more accurate statistical measure of the theoretical concept of diaschisis. However, in contrast to Salvalaggio and colleagues' (2020) work, we used the Disconnectome (Thiebaut de Schotten et al. 2020) developed on an independent and much larger cohort of stroke patients dataset $(n=1300)$ to characterise the profile of disconnection of individual patients. The Disconnectome allowed increasing the predictive power for motor impairments from $37 \%$ to $56 \%$ (right hemisphere lesion) and from $42 \%$ to $48 \%$ (left hemisphere lesion) at 2 weeks. These progresses are indicative of the importance of the disconnectome in the prediction of neuropsychological impairment and a significant step forward personalised prognosis in clinical neuropsychology.

In addition, it is worth noting that in the current study, we predict long-term symptoms at one year. A longterm prediction at one year based on acute brain imaging is challenging as additional factors may interact with the recovery (e.g., intensive rehabilitation, plasticity), which will have to be studied further before this work can be routinely incorporated into a clinical routine. For instance, in the current study, we observed a drop in the model's ability to capture the variance in right-sided motor scores of patients from $70 \%$ ( 2 weeks) to $21 \%$ ( 1 year). This might be due to the variability of brain plasticity capacities and functional coping strategies across patients. Additional factors such as, for example, patients' demographics (e.g. age, education) might increase the model's accuracy for long-term symptom predictions in future research.

It is worth noting that the model's performance does not drop in the testing dataset. For example, the model can capture $81 \%$ of the observed variability in the right-sided motor score at one year. In contrast, we observe $25 \%$ of explained variance by the same model for patients in the testing dataset. This result emphasises that models without external validation are not reliable for use in clinical practice because they cannot accurately predict individual motor outcomes of new patients. 
Studying motor impairments longitudinally allowed us to indirectly assess plasticity mechanisms over time. We demonstrated different patterns of disconnections responsible for motor impairments at two weeks, three months, and one year after a stroke. Particularly some components that were not initially responsible for the motor impairment (two weeks after the stroke) contribute later. This difference between the time points indicates that some disconnection related deficits can recover, but others are critical to long-term deficits (three month or one year). For instance, right sided motor deficits were well predicted by a disconnection of the left corticospinal tract at two weeks, followed by a widespread involvement of a bilateral fronto-parietal network at three months, and a focal contribution of sensorymotor interactions after one year. This result might be consistent with previous studies where, during the recovery of motor functions, the brain activity changed from widespread to focal cortical hyperexcitability (Ward et al. 2003; Bartolomeo and Thiebaut de Schotten 2016; Cramer 2008; Corbetta et al. 2005). Surprisingly, for right hemisphere lesions, the maximum contribution was not related to the corticospinal tract but to insular and frontotemporal connections. Damage to the insula, the precentral gyrus and frontal projections to the posterior cortex in the right hemisphere have been associated with hemispatial neglect (He et al. 2007; Corbetta et al. 2005; Corbetta and Shulman 2011; Doricchi et al. 2008; Lunven et al. 2015; Bartolomeo et al. 2007; Doricchi and Tomaiuolo 2003; Ying 2019; Jehkonen et al. 2006), which is a negative predictive factor of motor recovery, and disorders of body awareness, particularly anosognosia for hemiplegia, where internal emotional processes are not associated with bodily state signals from external sensory information (Pacella et al. 2019). Hence, it appears that left body motor impairments are not only due to a disconnection of the corticospinal tract but might tap into a wider network beyond pure motor functions. Furthermore, this apparent asymmetry in the critical circuitry leading to a motor impairment shows that hemispheric differences exist for motor behaviour, comparably to motor apraxia that was reported to have an asymmetrical neural bases (Liepmann 1925; Goldenberg 2003, 2008).

Although the current study allowed us to predict motor outcomes of patients at different time points after a stroke, the model based on hierarchical regression still requires improvements, when predicting longterm symptoms. We believe that adding other factors (e.g. demographic, clinical, socioeconomic variables) that likely interact with the recovery of patients can help us increase the model's predictive power. For example, there is evidence that a multimodal approach can outperform single-modality-based algorithms in the discrimination of patients (Lu et al. 2018). In this instance, different modalities complement and confirm each other and thus, this redundancy coming from various sources allows the algorithm to better estimate the prediction. Adding age, sex, brain volume, or resting-state functional MRI data as an additional modality, for example, could provide valuable information to the algorithm's prediction accuracy during training. Despite these advantages, multimodal prediction algorithms are rarely clinically feasible and often too expensive for large-scale longitudinal studies in terms of acquisition, computing powers, and analysis time. Further the purpose of this investigation was to assess the sole contribution of brain disconnection to the prediction of motor performance longitudinally after a stroke, not the contribution of other factors to build the model that best fits. 
Overall, we managed to increase the model's prediction quality two weeks after a stroke using the Disconnectome developed in an independent cohort of patients. This is a big step toward creating a clinical tool that will be able to complement prognosis of motor symptoms recovery in individual patients. We believe that using a multimodal approach in future studies (e.g. the inclusion of additional factors) and increasing the cohort size will allow the model to make more accurate long-term personalised predictions which, in turn, will inform tailored rehabilitation pathways. This achievement would only be possible within an open science framework capitalising on an effort to form collaborations between neurological centres to pool data.

\section{Declarations}

\section{Acknowledgments}

We thank the University of Bordeaux and CNRS for the infrastructural support. This project has received funding from the European Research Council (ERC) under the European Union's Horizon 2020 research and innovation programme (grant agreement No. 818521), the Marie Skłodowska-Curie programme (grant agreement No. 101028551), and The Erasmus+ for Traineeships Programme.

\section{Data availability}

The Hierarchical Regression Analysis with 5000 permutations was carried out in RStudio (v.1.3.1093; RStudio Team, 2020). The code is freely available (https://github.com/lidulyan/Hierarchical-LinearRegression-R-).

\section{References}

1. Aqueveque P, Ortega P, Pino E, Saavedra F, Germany E, Gómez B (2017) After Stroke Movement Impairments: A Review of Current Technologies for Rehabilitation. In: Tan U (ed) In Physical Disabilities - Therapeutic Implications. IntechOpen. doi:https://doi.org/10.5772/67577

2. Avants BB, Tustison NJ, Song G, Cook PA, Klein A, Gee JC (2011) A reproducible evaluation of ANTs similarity metric performance in brain image registration. Neurolmage 54(3):2033-2044. doi:10.1016/j.neuroimage.2010.09.025

3. Bartolomeo P, Thiebaut de Schotten M (2016) Let thy left brain know what thy right brain doeth: Interhemispheric compensation of functional deficits after brain damage. Neuropsychologia. doi:10.1016/j.neuropsychologia.2016.06.016

4. Bartolomeo P, Thiebaut de Schotten M, Doricchi F (2007) Left unilateral neglect as a disconnection syndrome. Cereb Cortex 17(11):2479-2490. doi:10.1093/cercor/bhl181

5. Bhuiyan PS, Bhuiyan PS, Rajgopal L, Shyamkishore K, ProQuest (2014) Inderbir SIngh's textbook of human neuroanatomy (fundamental and clinical). Ninth edition. edn. Jaypee Brothers Medical Publishers (P) Ltd, New Delhi, India 
6. Boyd LA, Hayward KS, Ward NS, Stinear CM, Rosso C, Fisher RJ, Carter AR, Leff AP, Copland DA, Carey LM, Cohen LG, Basso DM, Maguire JM, Cramer SC (2017) Biomarkers of stroke recovery: Consensus-based core recommendations from the Stroke Recovery and Rehabilitation Roundtable. International Journal of Stroke 12(5):480-493. doi:10.1177/1747493017714176

7. Brett M, Leff AP, Rorden C, Ashburner J (2001) Spatial Normalization of Brain Images with Focal Lesions Using Cost Function Masking. Neurolmage 14(2):486-500. doi:https://doi.org/10.1006/nimg.2001.0845

8. Carrera E, Tononi G (2014) Diaschisis: past, present, future. Brain 137(9):2408-2422. doi:10.1093/brain/awu101

9. Carter AR, Astafiev SV, Lang CE, Connor LT, Rengachary J, Strube MJ, Pope DL, Shulman GL, Corbetta M (2010) Resting interhemispheric functional magnetic resonance imaging connectivity predicts performance after stroke. Ann Neurol 67(3):365-375. doi:10.1002/ana.21905

10. Carter AR, Patel KR, Astafiev SV, Snyder AZ, Rengachary J, Strube MJ, Pope A, Shimony JS, Lang CE, Shulman GL, Corbetta M (2012) Upstream dysfunction of somatomotor functional connectivity after corticospinal damage in stroke. Neurorehabil Neural Repair 26(1):7-19.

doi:10.1177/1545968311411054

11. Catani M, ffytche $\mathrm{DH}(2005)$ The rises and falls of disconnection syndromes. Brain $128(\mathrm{Pt}$ 10):2224-2239. doi:10.1093/brain/awh622

12. Catani M, Thiebaut de Schotten $M$ (2008) A diffusion tensor imaging tractography atlas for virtual in vivo dissections. Cortex 44(8):1105-1132. doi:10.1016/j.cortex.2008.05.004

13. Catani M, Thiebaut de Schotten M (2012) Atlas of Human Brain Connections. Oxford University Press, Oxford

14. Cheung VCK, Turolla A, Agostini M, Silvoni S, Bennis C, Kasi P, Paganoni S, Bonato P, Bizzi E (2012) Muscle synergy patterns as physiological markers of motor cortical damage. Proceedings of the National Academy of Sciences 109 (36):14652. doi:10.1073/pnas.1212056109

15. Coleman ER, Moudgal R, Lang K, Hyacinth HI, Awosika OO, Kissela BM, Feng W (2017) Early Rehabilitation After Stroke: a Narrative Review. Curr Atheroscler Rep 19(12):59. doi:10.1007/s11883017-0686-6

16. Connell LA, Smith MC, Byblow WD, Stinear CM (2018) Implementing biomarkers to predict motor recovery after stroke. NeuroRehabilitation 43(1):41-50. doi:10.3233/NRE-172395

17. Corbetta M, Kincade MJ, Lewis C, Snyder AZ, Sapir A (2005) Neural basis and recovery of spatial attention deficits in spatial neglect. Nat Neurosci 8(11):1603-1610

18. Corbetta M, Ramsey L, Callejas A, Baldassarre A, Hacker CD, Siegel JS, Astafiev SV, Rengachary J, Zinn K, Lang CE, Connor LT, Fucetola R, Strube M, Carter AR, Shulman GL (2015) Common behavioral clusters and subcortical anatomy in stroke. Neuron 85(5):927-941.

doi:10.1016/j.neuron.2015.02.027

19. Corbetta M, Shulman GL (2011) Spatial neglect and attention networks. Annu Rev Neurosci 34:569599. doi:10.1146/annurev-neuro-061010-113731 
20. Cramer SC (2008) Repairing the human brain after stroke: I. Mechanisms of spontaneous recovery. Ann Neurol 63(3):272-287. doi:10.1002/ana.21393

21. Demeurisse G, Demol O, Robaye E (1980) Motor Evaluation in Vascular Hemiplegia. Eur Neurol 19(6):382-389. doi:10.1159/000115178

22. Doricchi F, Thiebaut de Schotten M, Tomaiuolo F, Bartolomeo P (2008) White matter (dis)connections and gray matter (dys)functions in visual neglect: Gaining insights into the brain networks of spatial awareness. Cortex 44(8):983-995. doi:10.1016/j.cortex.2008.03.006

23. Doricchi F, Tomaiuolo F (2003) The anatomy of neglect without hemianopia: a key role for parietalfrontal disconnection? NeuroReport 14(17):2239-2243. doi:10.1097/01.wnr.0000091132.75061.64

24. Dreeben $O$ (2008) Physical therapy clinical handbook for PTAs. Jones and Bartlett Publishers, Sudbury, Mass

25. Feigin VL, Stark BA, Johnson CO, Roth GA, Bisignano C, Abady GG, Abbasifard M, Abbasi-Kangevari M, Abd-Allah F, Abedi V, Abualhasan A, Abu-Rmeileh NME, Abushouk Al, Adebayo OM, Agarwal G, Agasthi P, Ahinkorah BO, Ahmad S, Ahmadi S, Ahmed Salih Y, Aji B, Akbarpour S,Akinyemi RO, Al Hamad H, Alahdab F, Alif SM, Alipour V, Aljunid SM, Almustanyir S,Al-Raddadi RM, Al-Shahi Salman R, Alvis-Guzman N, Ancuceanu R, Anderlini D, Anderson JA, Ansar A, Antonazzo IC, Arabloo J, Ärnlöv J, Artanti KD, Aryan Z, Asgari S, Ashraf T, Athar M, Atreya A, Ausloos M, Baig AA, Baltatu OC, Banach M, Barboza MA, Barker-Collo SL, Bärnighausen TW, Barone MTU, Basu S, Bazmandegan G, Beghi E, Beheshti M, Béjot Y, Bell AW, Bennett DA, Bensenor IM, Bezabhe WM, Bezabih YM, Bhagavathula AS, Bhardwaj P, Bhattacharyya K, Bijani A, Bikbov B, Birhanu MM, Boloor A, Bonny A, Brauer M, Brenner H, Bryazka D, Butt ZA, Caetano dos Santos FL, Campos-Nonato IR, Cantu-Brito C, Carrero JJ, CastañedaOrjuela CA, Catapano AL, Chakraborty PA, Charan J, Choudhari SG, Chowdhury EK, Chu D-T, Chung SC, Colozza D, Costa VM, Costanzo S, Criqui MH, Dadras O, Dagnew B, Dai X, Dalal K, Damasceno AAM, D'Amico E, Dandona L, Dandona R, Darega Gela J,Davletov K, De la Cruz-Góngora V, Desai R, Dhamnetiya D, Dharmaratne SD, Dhimal ML,Dhimal M, Diaz D, Dichgans M, Dokova K, Doshi R, Douiri A, Duncan BB, Eftekharzadeh S, Ekholuenetale M, El Nahas N, Elgendy IY, Elhadi M, El-Jaafary SI, Endres M, Endries AY, Erku DA, Faraon EJA, Farooque U, Farzadfar F, Feroze AH, Filip I, Fischer F, Flood D, Gad MM, Gaidhane S, Ghanei Gheshlagh R, Ghashghaee A, Ghith N, Ghozali G, Ghozy S, Gialluisi A, Giampaoli S, Gilani SA, Gill PS, Gnedovskaya EV, Golechha M, Goulart AC, Guo Y, Gupta R, Gupta VB, Gupta VK, Gyanwali P, Hafezi-Nejad N, Hamidi S, Hanif A, Hankey GJ, Hargono A, Hashi A, Hassan TS, Hassen HY, Havmoeller RJ, Hay SI, Hayat K, Hegazy MI, Herteliu C, Holla R, Hostiuc S, Househ M, Huang J, Humayun A, Hwang B-F, lacoviello L, lavicoli I, Ibitoye SE, llesanmi OS, llic IM, llic MD, Iqbal U,Irvani SSN, Islam SMS, Ismail NE, Iso H, Isola G, Iwagami M, Jacob L, Jain V, Jang S-I, Jayapal SK, Jayaram S, Jayawardena R, Jeemon P, Jha RP, Johnson WD, Jonas JB, Joseph N, Jozwiak JJ, Jürisson M, Kalani R, Kalhor R, Kalkonde Y, Kamath A, Kamiab Z, Kanchan T, Kandel H, Karch A, Katoto PDMC, Kayode GA, Keshavarz P, Khader YS, Khan EA, Khan IA, Khan M, Khan MAB, Khatib MN, Khubchandani J, Kim GR, Kim MS, Kim YJ,Kisa A, Kisa S, Kivimäki M, Kolte D, Koolivand A, Koulmane Laxminarayana SL, Koyanagi A, Krishan K, Krishnamoorthy V, Krishnamurthi RV, Kumar GA, Kusuma D, La Vecchia C,Lacey B, Lak HM, Lallukka T, Lasrado S, Lavados PM, Leonardi M, Li B, 
Li S, Lin H,Lin R-T, Liu X, Lo WD, Lorkowski S, Lucchetti G, Lutzky Saute R, Magdy Abd El Razek H, Magnani FG, Mahajan PB, Majeed A, Makki A, Malekzadeh R, Malik AA, Manafi N, Mansournia MA, Mantovani LG, Martini S, Mazzaglia G, Mehndiratta MM, Menezes RG, Meretoja A,Mersha AG, Miao Jonasson J, Miazgowski B, Miazgowski T, Michalek IM, Mirrakhimov EM,Mohammad Y, Mohammadian-Hafshejani A, Mohammed S, Mokdad AH, Mokhayeri Y, Molokhia M, Moni MA, Montasir AA, Moradzadeh R, Morawska L, Morze J, Muruet W, Musa KI, Nagarajan AJ, Naghavi M, Narasimha Swamy S, Nascimento BR, Negoi RI, Neupane Kandel S, Nguyen TH, Norrving B, Noubiap JJ, Nwatah VE, Oancea B, Odukoya OO, Olagunju AT, Orru H,Owolabi MO, Padubidri JR, Pana A, Parekh T, Park E-C, Pashazadeh Kan F, Pathak M,Peres MFP, Perianayagam A, Pham T-M, Piradov MA, Podder V, Polinder S, Postma MJ,Pourshams A, Radfar A, Rafiei A, Raggi A, Rahim F, RahimiMovaghar V, Rahman M, Rahman MA, Rahmani AM, Rajai N, Ranasinghe P, Rao CR, Rao SJ, Rathi P, Rawaf DL, Rawaf S, Reitsma MB, Renjith V, Renzaho AMN, Rezapour A, Rodriguez JAB, Roever L, Romoli M,Rynkiewicz A, Sacco S, Sadeghi M, Saeedi Moghaddam S, Sahebkar A, Saif-Ur-Rahman KM,Salah R, Samaei M, Samy AM, Santos IS, Santric-Milicevic MM, Sarrafzadegan N, Sathian B, Sattin D, Schiavolin S, Schlaich MP, Schmidt MI, Schutte AE, Sepanlou SG, Seylani A, Sha F, Shahabi S, Shaikh MA, Shannawaz M, Shawon MSR, Sheikh A, Sheikhbahaei S,Shibuya K, Siabani S, Silva DAS, Singh JA, Singh JK, Skryabin VY, Skryabina AA, Sobaih BH, Stortecky S, Stranges S, Tadesse EG, Tarigan IU, Temsah M-H, Teuschl Y, Thrift AG, Tonelli M, Tovani-Palone MR, Tran BX, Tripathi M, Tsegaye GW, Ullah A, Unim B,Unnikrishnan B, Vakilian A, Valadan Tahbaz S, Vasankari TJ, Venketasubramanian N,Vervoort D, Vo B, Volovici V, Vosoughi K, Vu GT, Vu LG, Wafa HA, Waheed Y, Wang Y,Wijeratne T, Winkler AS, Wolfe CDA, Woodward M, Wu JH, Wulf Hanson S, Xu X, Yadav L, Yadollahpour A, Yahyazadeh Jabbari SH, Yamagishi K, Yatsuya H, Yonemoto N, Yu C,Yunusa I, Zaman MS, Zaman SB, Zamanian M, Zand R, Zandifar A, Zastrozhin MS, Zastrozhina A, Zhang Y, Zhang Z-J, Zhong C, Zuniga YMH, Murray CJL (2021) Global, regional, and national burden of stroke and its risk factors, 1990-2019: a systematic analysis for the Global Burden of Disease Study 2019. The Lancet Neurology 20 (10):795-820.doi:10.1016/S1474-4422(21)00252-0

26. Fess E, Moran C (1981) American Society of Hand Therapists Clinical Assessment Recommendations

27. Feys H, Van Hees J, Bruyninckx F, Mercelis R, De Weerdt W (2000) Value of somatosensory and motor evoked potentials in predicting arm recovery after a stroke. J Neurol Neurosurg Psychiatry 68(3):323-331. doi:10.1136/jnnp.68.3.323

28. Finger S, Koehler PJ, Jagella C (2004) The Monakow concept of diaschisis: origins and perspectives. Arch Neurol 61(2):283-288. doi:10.1001/archneur.61.2.283

29. Forkel SJ, Friedrich P, Thiebaut de Schotten M, Howells H (2021) White matter variability, cognition, and disorders: a systematic review. Brain Structure and Function. doi:10.1007/s00429-021-02382-w

30. Foulon C, Cerliani L, Kinkingnehun S, Levy R, Rosso C, Urbanski M, Volle E, Thiebaut de Schotten M (2018) Advanced lesion symptom mapping analyses and implementation as BCBtoolkit. Gigascience 7(3):1-17. doi:10.1093/gigascience/giy004 
31. Glasser MF, Coalson TS, Robinson EC, Hacker CD, Harwell J, Yacoub E, Ugurbil K, Andersson J, Beckmann CF, Jenkinson M, Smith SM, Van Essen DC (2016) A multi-modal parcellation of human cerebral cortex. Nature 536(7615):171-178. doi:10.1038/nature18933

32. Goldenberg G (2003) Apraxia and beyond: life and work of Hugo Liepmann. Cortex 39(3):509-524

33. Goldenberg G (2008) Apraxia and the parietal lobes. Neuropsychologia. doi:10.1016/j.neuropsychologia.2008.07.014

34. Grabner G, Janke AL, Budge MM, Smith D, Pruessner J, Collins DL (2006) Symmetric Atlasing and Model Based Segmentation: An Application to the Hippocampus in Older Adults. In: Larsen R, Nielsen M, Sporring J (eds) Medical Image Computing and Computer-Assisted Intervention - MICCAI 2006, Berlin, Heidelberg, // 2006. Springer Berlin Heidelberg, pp 58-66

35. Granziera C, Daducci A, Meskaldji DE, Roche A, Maeder P, Michel P, Hadjikhani N, Sorensen AG, Frackowiak RS, Thiran JP, Meuli R, Krueger G (2012) A new early and automated MRI-based predictor of motor improvement after stroke. Neurology 79(1):39-46. doi:10.1212/WNL.0b013e31825f25e7

36. Gurol ME, Kim JS (2018) Advances in Stroke Prevention in 2018. J Stroke 20(2):143-144. doi:10.5853/jos.2018.01438

37. Hatem SM, Saussez G, della Faille M, Prist V, Zhang X, Dispa D, Bleyenheuft Y (2016) Rehabilitation of Motor Function after Stroke: A Multiple Systematic Review Focused on Techniques to Stimulate Upper Extremity Recovery. Front Hum Neurosci 10(442). doi:10.3389/fnhum.2016.00442

38. He BJ, Snyder A, Vincent JL, Epstein A, Shulman GL, Corbetta M (2007) Breakdown of functional connectivity in frontoparietal networks underlies behavioral deficits in spatial neglect. Neuron 53(6):905-918. doi:10.1016/j.neuron.2007.02.013

39. Heil BJ, Hoffman MM, Markowetz F et al (2021) Reproducibility standards for machine learning in the life sciences. Nat Methods 18:1132-1135. https://doi.org/10.1038/s41592-021-01256-7

40. Hope TMH, Seghier ML, Prejawa S, Leff AP, Price CJ (2016) Distinguishing the effect of lesion load from tract disconnection in the arcuate and uncinate fasciculi. Neurolmage 125:1169-1173. doi:10.1016/j.neuroimage.2015.09.025

41. Jehkonen M, Laihosalo M, Kettunen JE (2006) Impact of neglect on functional outcome after stroke: a review of methodological issues and recent research findings. Restor Neurol Neurosci 24(46):209-215

42. Kelly K, Brander F, Strawson A, Ward N, Hayward K (2020) Pushing the limits of recovery in chronic stroke survivors: a descriptive qualitative study of users perceptions of the Queen Square Upper Limb Neurorehabilitation Programme. BMJ Open 10(10):e036481. doi:10.1136/bmjopen-2019-036481

43. Kempen JC, de Groot V, Knol DL, Polman CH, Lankhorst GJ, Beckerman H (2011) Community walking can be assessed using a 10-metre timed walk test. Mult Scler 17(8):980-990. doi:10.1177/1352458511403641

44. Kim B, Winstein C (2017) Can Neurological Biomarkers of Brain Impairment Be Used to Predict Poststroke Motor Recovery? A Systematic Review. Neurorehabil Neural Repair 31(1):3-24. doi:10.1177/1545968316662708 
45. Klein A, Andersson J, Ardekani BA, Ashburner J, Avants B, Chiang MC, Christensen GE, Collins DL, Gee J, Hellier P, Song JH, Jenkinson M, Lepage C, Rueckert D, Thompson P, Vercauteren T, Woods RP, Mann JJ, Parsey RV (2009) Evaluation of 14 nonlinear deformation algorithms applied to human brain MRI registration. Neurolmage 46(3):786-802. doi:10.1016/j.neuroimage.2008.12.037

46. Koch PJ, Park C-H, Girard G, Beanato E, Egger P, Evangelista GG, Lee J, Wessel MJ, Morishita T, Koch G, Thiran J-P, Guggisberg AG, Rosso C, Kim Y-H, Hummel FC (2021) The structural connectome and motor recovery after stroke: predicting natural recovery. Brain 144(7):2107-2119. doi:10.1093/brain/awab082

47. Kuceyeski A, Maruta J, Relkin N, Raj A (2013) The Network Modification (NeMo) Tool: elucidating the effect of white matter integrity changes on cortical and subcortical structural connectivity. Brain Connect 3(5):451-463. doi:10.1089/brain.2013.0147

48. Kusec A, Velikonja D, DeMatteo C, Harris JE (2019) Motivation in rehabilitation and acquired brain injury: can theory help us understand it? Disabil Rehabil 41(19):2343-2349. doi:10.1080/09638288.2018.1467504

49. Liepmann H (1925) Apraktishe Störungen.. In: In: Kramer IHCaF (ed) Lehrbuch der Nervenkrakheiten. Springer, Berlin, pp 408-416

50. Lin G, Zhuang C, Shen Z, Xiao G, Chen Y, Shen Y, Zong X, Wu R (2018) APT Weighted MRI as an Effective Imaging Protocol to Predict Clinical Outcome After Acute Ischemic Stroke. Front Neurol 9:901. doi:10.3389/fneur.2018.00901

51. Lu D, Popuri K, Ding GW, Balachandar R, Beg MF, Alzheimer's Disease Neuroimaging I (2018) Multimodal and Multiscale Deep Neural Networks for the Early Diagnosis of Alzheimer's Disease using structural MR and FDG-PET images. Sci Rep 8(1):5697. doi:10.1038/s41598-018-22871-z

52. Lunven M, Thiebaut De Schotten M, Bourlon C, Duret C, Migliaccio R, Rode G, Bartolomeo P (2015) White matter lesional predictors of chronic visual neglect: a longitudinal study. Brain 138(Pt 3):746760. doi:10.1093/brain/awu389

53. Lyle RC (1981) A performance test for assessment of upper limb function in physical rehabilitation treatment and research.International Journal of Rehabilitation Research4 (4)

54. Mathiowetz V, Weber K, Kashman N, Volland G (1985) Adult Norms for the Nine Hole Peg Test of Finger Dexterity. The Occupational Therapy Journal of Research 5(1):24-38. doi:10.1177/153944928500500102

55. Moura LM, Luccas R, de Paiva JPQ, Amaro E Jr, Leemans A, Leite CDC, Otaduy MCG, Conforto AB (2019) Diffusion Tensor Imaging Biomarkers to Predict Motor Outcomes in Stroke: A Narrative Review. Front Neurol 10:445. doi:10.3389/fneur.2019.00445

56. Nachev P, Coulthard E, Jager HR, Kennard C, Husain M (2008) Enantiomorphic normalization of focally lesioned brains. Neurolmage 39(3):1215-1226. doi:10.1016/j.neuroimage.2007.10.002

57. Nijland RH, van Wegen EE, Harmeling-van der Wel BC, Kwakkel G, Investigators E (2010) Presence of finger extension and shoulder abduction within 72 hours after stroke predicts functional recovery: 
early prediction of functional outcome after stroke: the EPOS cohort study. Stroke 41(4):745-750. doi:10.1161/STROKEAHA.109.572065

58. Oxford Grice K, Vogel KA, Le V, Mitchell A, Muniz S, Vollmer MA (2003) Adult Norms for a Commercially Available Nine Hole Peg Test for Finger Dexterity. The American Journal of Occupational Therapy 57(5):570-573. doi:10.5014/ajot.57.5.570

59. Pacella V, Foulon C, Jenkinson PM, Scandola M, Bertagnoli S, Avesani R, Fotopoulou A, Moro V, de Thiebaut M (2019) Anosognosia for hemiplegia as a tripartite disconnection syndrome. Elife 8. doi:10.7554/eLife.46075

60. Perry J, Garrett M, Gronley JK, Mulroy SJ (1995) Classification of walking handicap in the stroke population. Stroke 26(6):982-989. doi:10.1161/01.str.26.6.982

61. Portnov BA, Dubnov J, Barchana M (2007) On ecological fallacy, assessment errors stemming from misguided variable selection, and the effect of aggregation on the outcome of epidemiological study. J Expo Sci Environ Epidemiol 17(1):106-121. doi:10.1038/sj.jes.7500533

62. Puig J, Pedraza S, Blasco G, Daunis IEJ, Prados F, Remollo S, Prats-Galino A, Soria G, Boada I, Castellanos M, Serena J (2011) Acute damage to the posterior limb of the internal capsule on diffusion tensor tractography as an early imaging predictor of motor outcome after stroke. AJNR Am J Neuroradiol 32(5):857-863. doi:10.3174/ajnr.A2400

63. Rehme AK, Eickhoff SB, Wang LE, Fink GR, Grefkes C (2011) Dynamic causal modeling of cortical activity from the acute to the chronic stage after stroke. Neurolmage 55(3):1147-1158. doi:10.1016/j.neuroimage.2011.01.014

64. Ripolles P, Marco-Pallares J, de Diego-Balaguer R, Miro J, Falip M, Juncadella M, Rubio F, RodriguezFornells A (2012) Analysis of automated methods for spatial normalization of lesioned brains. Neurolmage 60(2):1296-1306. doi:10.1016/j.neuroimage.2012.01.094

65. Robinson WS (1950) Ecological Correlations and the Behavior of Individuals. Am Sociol Rev 15(3):351-357. doi: $10.2307 / 2087176$

66. Rojkova K, Volle E, Urbanski M, Humbert F, Dell'Acqua F, Thiebaut de Schotten M (2016) Atlasing the frontal lobe connections and their variability due to age and education: a spherical deconvolution tractography study. Brain Struct Funct 221(3):1751-1766. doi:10.1007/s00429-015-1001-3

67. Salvalaggio A, De Filippo De Grazia M, Zorzi M, Thiebaut de Schotten M, Corbetta M (2020) Poststroke deficit prediction from lesion and indirect structural and functional disconnection. Brain 143(7):2173-2188. doi:10.1093/brain/awaa156

68. Schiemanck SK, Kwakkel G, Post MW, Kappelle LJ, Prevo AJ (2006) Predicting long-term independency in activities of daily living after middle cerebral artery stroke: does information from MRI have added predictive value compared with clinical information? Stroke 37(4):1050-1054. doi:10.1161/01.STR.0000206462.09410.6f

69. Smith S, Duff E, Groves A, Nichols TE, Jbabdi S, Westlye LT, Tamnes CK, Engvig A, Walhovd KB, Fjell AM, Johansen-Berg H, Douaud G (2019) Structural Variability in the Human Brain Reflects Fine- 
Grained Functional Architecture at the Population Level. J Neurosci 39(31):6136-6149.

doi:10.1523/JNEUROSCI.2912-18.2019

70. Stinear CM (2017) Prediction of motor recovery after stroke: advances in biomarkers. Lancet Neurol 16(10):826-836. doi:10.1016/S1474-4422(17)30283-1

71. Thiebaut de Schotten M, Dell'Acqua F, Ratiu P, Leslie A, Howells H, Cabanis E, Iba-Zizen MT, Plaisant O, Simmons A, Dronkers NF, Corkin S, Catani M (2015) From Phineas Gage and Monsieur Leborgne to H.M.: Revisiting Disconnection Syndromes. Cereb Cortex 25(12):4812-4827.

doi:10.1093/cercor/bhv173

72. Thiebaut de Schotten M, Foulon C (2018) The rise of a new associationist school for lesion-symptom mapping. Brain 141(1):2-4. doi:10.1093/brain/awx332

73. Thiebaut de Schotten M, Foulon C, Nachev P (2020) Brain disconnections link structural connectivity with function and behaviour. Nat Commun 11(1):5094. doi:10.1038/s41467-020-18920-9

74. Thiebaut de Schotten M, Kinkingnehun S, Delmaire C, Lehericy S, Duffau H, Thivard L, Volle E, Levy R, Dubois B, Bartolomeo P (2008) Visualization of disconnection syndromes in humans. Cortex 44(8):1097-1103. doi:10.1016/j.cortex.2008.02.003

75. Thiebaut de Schotten M, Tomaiuolo F, Aiello M, Merola S, Silvetti M, Lecce F, Bartolomeo P, Doricchi F (2014) Damage to White Matter Pathways in Subacute and Chronic Spatial Neglect: A Group Study and 2 Single-Case Studies with Complete Virtual “In Vivo” Tractography Dissection. Cereb Cortex 24(3):691-706. doi:10.1093/cercor/bhs351

76. Thiel A, Vahdat S (2015) Structural and resting-state brain connectivity of motor networks after stroke. Stroke 46(1):296-301. doi:10.1161/STROKEAHA.114.006307

77. Tidoni E, Tieri G, Aglioti SM (2015) Re-establishing the disrupted sensorimotor loop in deafferented and deefferented people: The case of spinal cord injuries. Neuropsychologia 79:301-309. doi:https://doi.org/10.1016/j.neuropsychologia.2015.06.029

78. van der Lee JH, Beckerman H, Lankhorst GJ, Bouter LM (2001) The responsiveness of the Action Research Arm test and the Fugl-Meyer Assessment scale in chronic stroke patients. J Rehabil Med 33(3):110-113. doi:10.1080/165019701750165916

79. Volle E, Levy R, Burgess P (2013) A new era for lesion-behavior mapping of prefrontal functions.. In. pp 500-523.. doi:10.13140/2.1.4509.0243

80. von Monakow C (1914) Die Lokalisation im Grosshirn und der Funktion durch kortikale Herde. Verlag von J.F. Bergmann, Wiesbaden

81. Vu AT, Auerbach E, Lenglet C, Moeller S, Sotiropoulos SN, Jbabdi S, Andersson J, Yacoub E, Ugurbil K (2015) High resolution whole brain diffusion imaging at 7T for the Human Connectome Project. Neurolmage 122:318-331. doi:10.1016/j.neuroimage.2015.08.004

82. Ward NS, Brown MM, Thompson AJ, Frackowiak RS (2003) Neural correlates of motor recovery after stroke: a longitudinal fMRI study. Brain 126(Pt 11):2476-2496. doi:10.1093/brain/awg245

83. Xu T, Rolf Jager H, Husain M, Rees G, Nachev P (2018) High-dimensional therapeutic inference in the focally damaged human brain. Brain 141(1):48-54. doi:10.1093/brain/awx288 
84. Ying X (2019) An Overview of Overfitting and its Solutions. Journal of Physics: Conference Series 1168:022022. doi:10.1088/1742-6596/1168/2/022022

\section{Figures}

\section{Figure 1}

Distribution of (a) lesions and (b) disconnection maps in 62 stroke patients in the MNI152 space ( $\mathrm{Z}$ coordinate indicated on each slice, neurological view: left=left). The colour bars represent a) the number of overlapping lesions and $b$ ) the probability of disconnection. L: left hemisphere; $n$ : number.

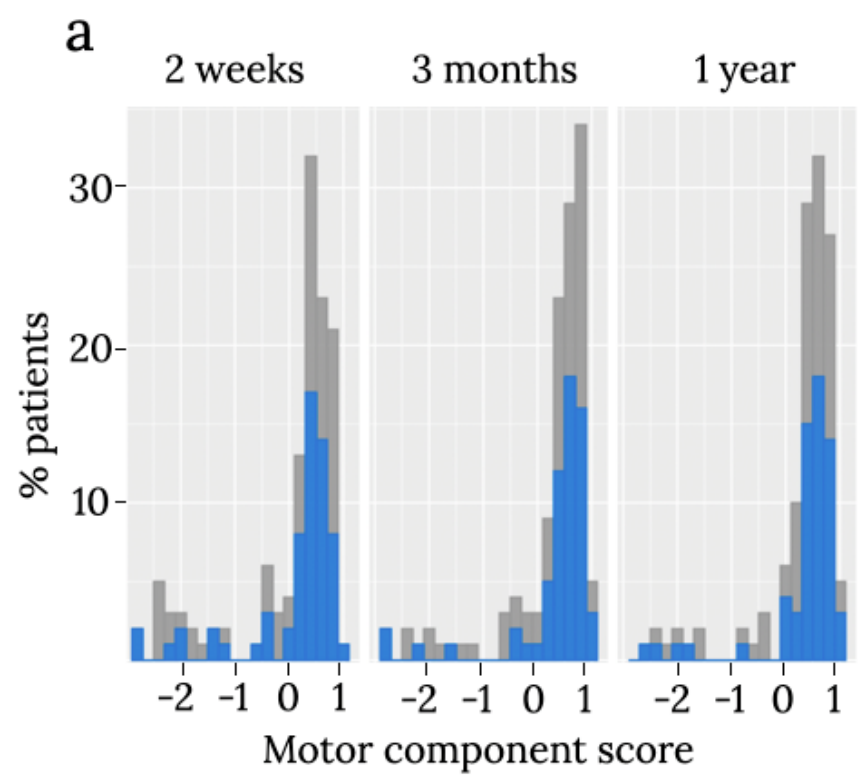

b

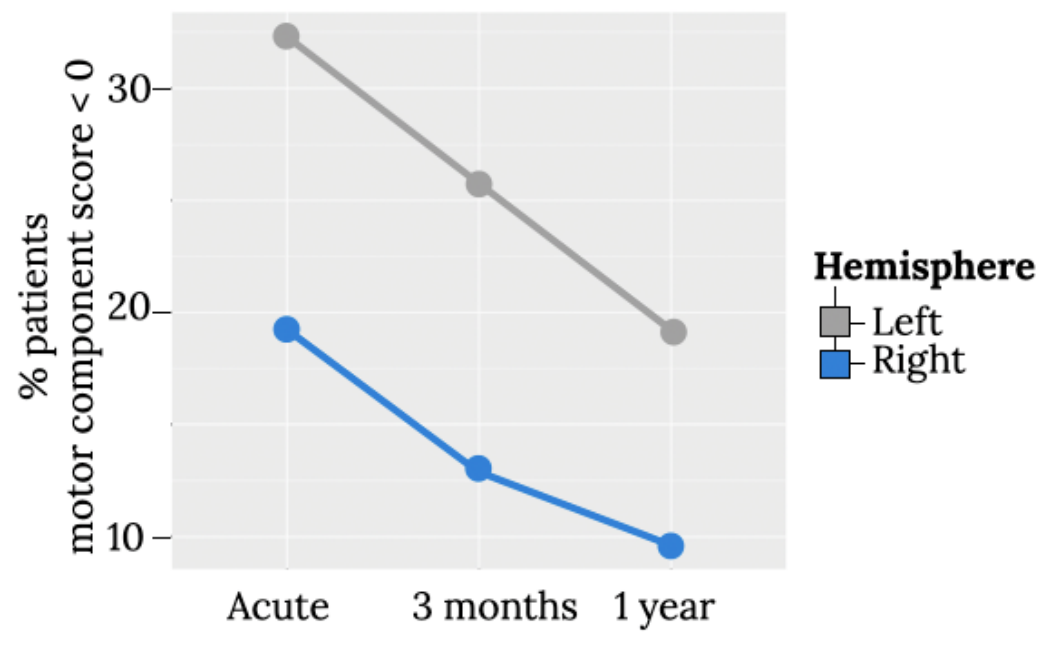

\section{Figure 2}

The distribution of motor scores across two weeks, three months, and one-year periods after stroke. a) Overall distribution of the score, b) Proportion of patients with a motor score $<0$. 


\section{Left motor component prediction}

n

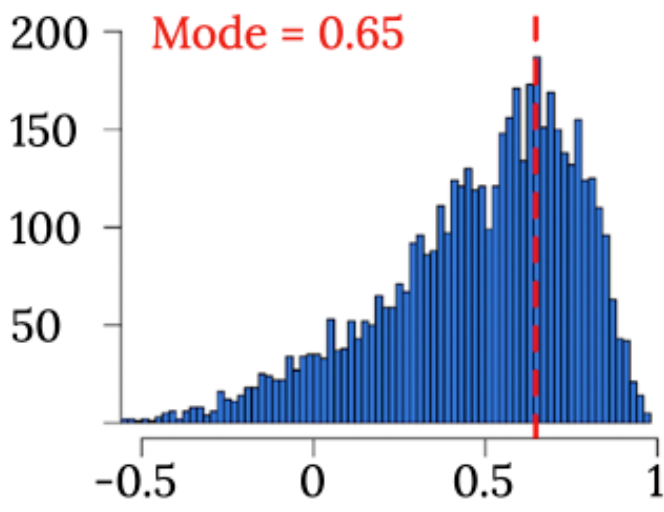

3 months

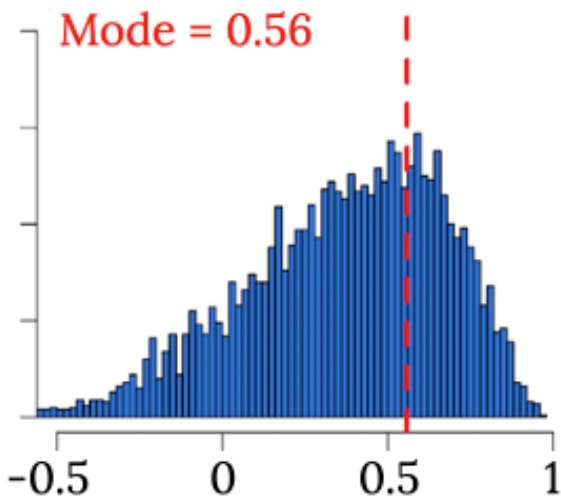

1 year

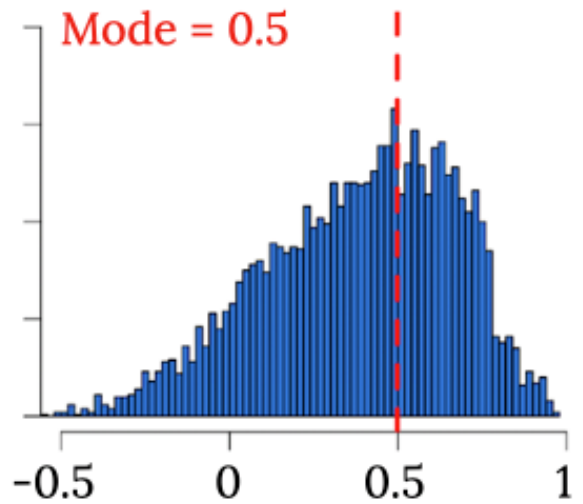

Right motor component prediction

n

2 weeks

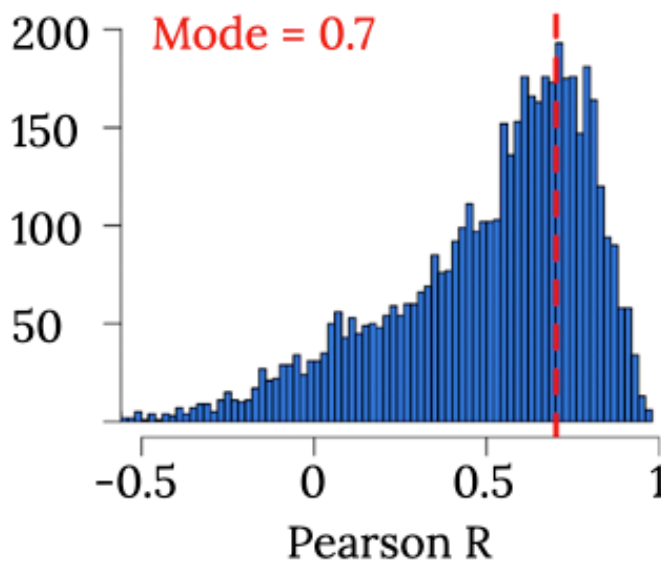

3 months

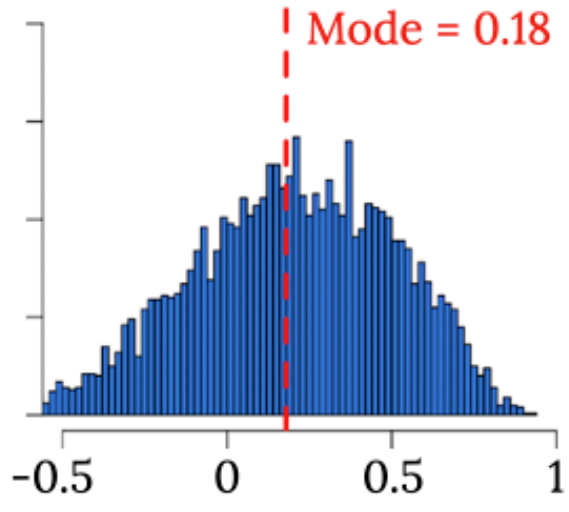

Pearson R
1 year

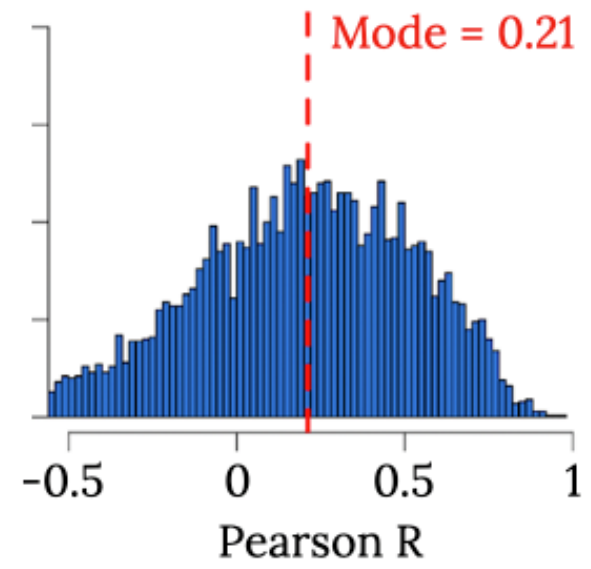

Figure 3

Histograms (5000 iterations in blue) of the prediction for motor scores at 2 weeks, three months, and oneyear after stroke for unseen data. The dashed red line indicates the mode of each histogram. n: number of iterations.

\section{Figure 4}

Prediction accuracy of the models of the hierarchical linear regression analysis in the a) training set (78\% of the data) and $b$ ) testing set ( $22 \%$ of the data), based on the mode of $\mathrm{R}$ distribution. Left and right indicate motor scores. 


\section{Left motor prediction}

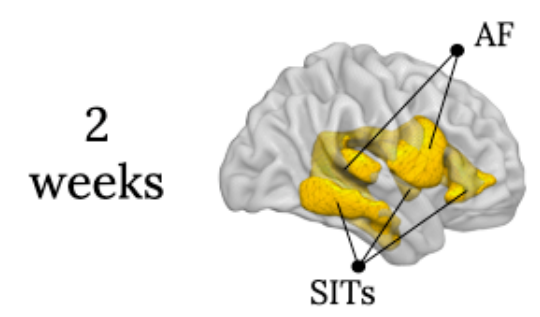

3 months

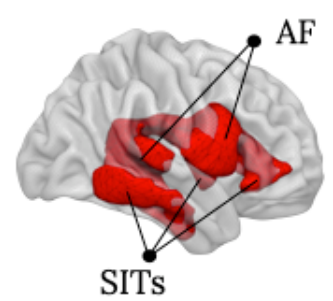

1 year
Right motor prediction
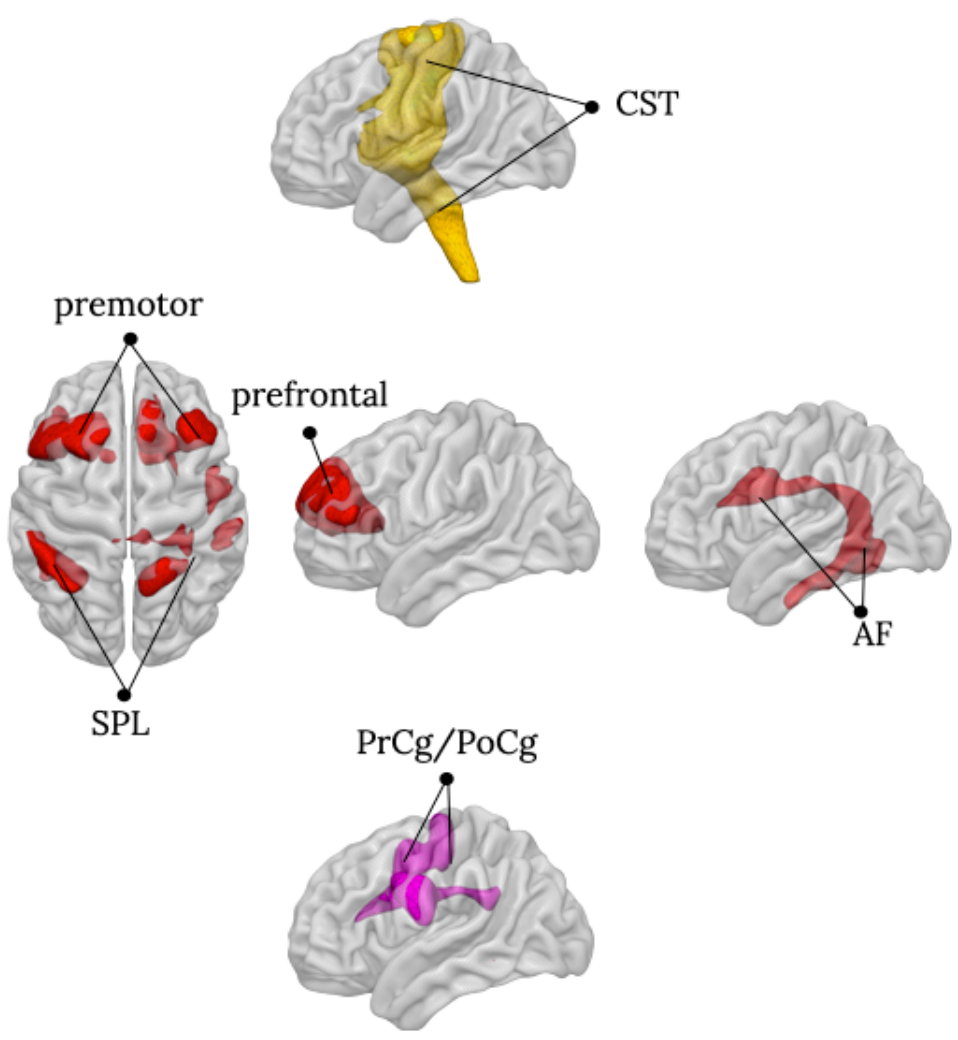

Figure 5

Top contributing components to the hierarchical regression $(p<0.001)$ at three time points after stroke onset (two weeks, three months, and one year) for motor impairments. 2W: two weeks after lesion onset, 3M: three months after lesion onset, 1Y: one year after lesion onset, AF: Arcuate Fasciculus, SITS: Short Insular Tracts, SMg: supramarginal gyrus white matter, SPL: Superior Parietal Lobule white matter, PrCg/PoCg: precentral/postcentral U-shaped fibres, CST: Cortico-Spinal Tract.

\section{Supplementary Files}

This is a list of supplementary files associated with this preprint. Click to download.

- SupplementaryOnlinematerial.pdf 